\title{
Remote sensing based on time variance control in configurable area partitioning
}

\author{
Stefano De Falco *, Giulia Fiorentino \\ Dept. of Political Sciences, University Federico II of Naples, Italy, sdefalco@unina.it. \\ * Corresponding author
}

\begin{abstract}
In this paper a sensor data fusion approach for characteristics field monitoring, based on time variance control model, is proposed. Distributed sensing and remote processing are the basic features of the employed architecture. In fact, in order to obtain meaningful information about the temporal and spatial variations, which characterize the field levels of some characteristics (electromagnetic, air pollution, seismic, etc), a distributed network of wireless and mobile smartsensors has been designed.
\end{abstract}

Starting from the partitioned configuration of a monitored geographic areas, this model allows to take into account the different levels of degradation over time in the sensors' performances associated with the different geographic partitions, progressively increasing the severity of the control. To this end, through the introduction of a reliability curve, a revised traditional control chart for variables is proposed.

The proposed approach, further constituting an element of the scientific debate, aims to be a useful operational tool for professionals and managers employed in the environment control.

Keywords: Remote sensing; monitoring; time variance.

\section{Introduction}

The main objective of monitoring is to obtain a better understanding of the observed phenomenon, combining a geographical approach with a metrological one. This task is not always easy, in fact, often a direct physical knowledge of the process behaviour or an 'a priori' information is not available, so should be done only generic assumptions. Therefore, a careful study is required in order to single out both influence factors and phenomenology correlated to the considered application.

The proposed model, starting from the partitioned configuration of a monitored geographic area (De Capua et al., 2005), allows to take into account the different levels of degradation over time in the performance of the sensors associated with the different geographic partitions, progressively increasing the severity of the control of the monitored parameters. To this end, through the introduction of a reliability curve, a review of the traditional control chart for variables is proposed. A similar approach based on reliability applied to the field of remote sensing in the energy sector is followed by Zhou and other authors (Zhou et alk., 2010). Other methods applied in different field are, for instance recognizable in monitoring the main vegetation changes (Favretto, 2018). Particularly, the basic idea behind the work is to consider the traditional tool for controlling environmental processes (Haibin et al., 2017) that insist on a certain geographical area, i.e. the variable control chart, and to modify this indicator in order to improve the quality of information that it produces. To modify it in this sense, this methodology takes into account a natural degradation over time of the performance of the sensors through which the processes are monitored.

Traditional control charts are based on the assumption that the measurement data detected by the field sensors, relating to the average of each subgroup of the parameters under observation of a monitored environmental characteristic, are reported regardless of the stability of its parameters over time (Elhoseny et al., 2015; Quing et al., 2006).

The traditional approach involves the construction of control charts characterized by stationary limits, ignoring the possibility of an "adjustment" of the aforementioned limits in relation to time (degradation after a certain delta t) and space (in terms of gradient between geographical partitions of a large area to be monitored). This conceptual innovation determines the possibility of having an instrument capable of carrying out a control of the "trajectory tracking" type, modulated on the possible variations of the measurand. The modeling for the described scenario is based on the implementation of the reliability function as a source of the variability of the control limits, as it can be predetermined through the life curves of the performance of the environmental sensors.

The results achievable through the proposed approach allow to improve the "sensitivity" of the remote monitoring sensor networks through the modulation of the control limits. In this way is, therefore, possible acquire a beneficial discriminatory power capable of separating the effects of random variability deriving from common 
causes, from those related to deterministic variability of the measurand.

The quality of the information collected through remote sensing is expressed with characteristics that can be verified with deterministic and probabilistic techniques. The formers are represented by tolerance limits and the measurement usually takes place by verifying their degree of conformity. The seconds are represented by expected performances over time of the monitored environmental characteristic.

These methodologies are used to guarantee the variation within allowed limits of environmental characteristics (EM field values, air pollution, etc.) over time and to prevent their associated human, natural or artificial risks (Chang, Yu, 2012).

The control charts could be used, in fact, to control the variability of the environmental characteristics to which man and the environment itself are exposed.

The variability of a monitored feature is affected by two different types of causes, common causes and special causes. The common causes are attributable to an identifiable set of anomalies and dysfunctions always present in a process. The variability depending from these causes is predictable: the "bell curve", representing the standard variability, is contained within standard limits. Special causes occur accidentally and induce systematic variations in the monitored environmental characteristic.

In the following paragraph the model of the revised control chart is developed considering the degradation of the environmental sensors' performance among the common causes. This solution allows a more severe control as it is more selective with respect to the intrinsic variation of the phenomena monitored in a geographical area. Subsequently, the results of an application of this controlmap to a real case of environmental monitoring will be reported and discussed. The conclusions then will close the contribution.

\section{Time Variance Control model}

In the traditional process control of an environmental characteristic by means of remote sensing, the control ranges normally associated with common causes are assumed according to the normal standard quantiles (figure 1), according to which (Huitian, 2001):

- $68.27 \%$ of the results of a measurement fall within the range $\mathrm{M} \pm \sigma$;

- $95.45 \%$ of the results of a measurement fall within the range $M \pm 2 \sigma$;

- $99.73 \%$ of the results of a measurement fall within the range $M \pm 3 \sigma$;

$$
\sigma=\frac{\sqrt{\sum_{i-1}^{N}\left(x_{i}-\bar{x}\right)^{2}}}{N-1}
$$

with $\sigma$ mean square deviation.

In this framework, a key point emerges concerning the fact that any environmental characteristic to be monitored, if considered isolated from any external influence, results characterized by its own natural variability that is generally adequately predictable.

A system under these conditions is in a state of control.

As previously mentioned, in this situation the system constituted by the characteristic to be monitored and by the network of remote sensors, comes to be influenced by a series of common causes:

- the common causes depend by the way in which the system was designed and built; these causes are, therefore, intrinsic features of the system;

- in the presence of only common causes, the extent of data dispersion is constant and predictable, and in this interval the variability is random;

- if only common causes act on a system, it makes no sense to operate on a specific aspect of the system, cause if the variability is too great it can only be reduced by modifying the system.

External causes outside of the system that create variability characterized by the same sign, are called special causes. These causes alter natural variability and generate unpredictable variability that disturbs the functioning of the process.

Unlike common causes, it is possible to act directly on special causes as these are outside the natural functioning of the system; in this situation it is necessary to act on a specific aspect of the system to remove the source of disturb and so consequently remove the variation it has generated.

The Control Chart is a statistical tool that allows to alert if a system is out of control. The most common form of the control chart records in chronological order and graphically the values detected by the sensors, with reference to limits that take into account the natural variability of the monitored process.

There are two basic types of control charts:

-variables' charts, used when it is necessary to take a measurable environmental characteristic under control (E.M fields, air pollution, etc);

-attributes' charts, used when the characteristic (and their values) should be judged in terms of "good" or "not good". The most common type of variable control chart is the X$\mathrm{R}$ chart.

The core values of the X-R Control Chart are:

- the average of the parameter $\mathrm{x}$, obtained from measurements performed on samples taken from an environment process to be monitored;

- the Range (R), representing the difference between the minimum and maximum values in the acquired samples;

- the upper and lower limits within which the process is maintained if it respects its natural variability.

Each point of the control chart reflects the average value of several samples of the same parameter. When a point gets out of bounds it is likely that the process is no longer under control.

The out of control state can also occur if the points do not remain outside the limits but they draw particular 
configurations inside the control limits not attributable to a random situation.

The X-R maps are presented as two distinct diagrams, which have in common the abscissa scale, graduated according to the frequency of the acquired samples deriving from field sensors, and show in ordinate data obtained from measurements carried out on the average values related to samples of the subgroups.

For the construction of X-R chart, could be traced: for the X Chart

- a continuous line corresponding to the average value $\mathrm{x}$, calculated as the average of the xi measured values, relating to the various subgroups considered, or of the expected average value, $\mathrm{x}$;

- two broken lines for process analysis, called Upper Control Limit (UCL) and Lower Control Limit (LCL);

- two dotted lines that identify the warning values that foreshadow the approach of out-of-control situations; for the R chart

- a continuous line corresponding to the $\mathrm{R}$ value, the average of the values of the deviation $R$ of all the considered subgroups;

- a broken line corresponding to the Upper Control Limit.

The distance between UCL and LCL from the mean line is established in relation to the permissible dispersion for the process according to the choice of severity level control compliance with the mission critical of the phenomena.

In a normal distribution, the probability that any value of the parameter under observation is included in the area under the curve, between the $\mathrm{x}-3 \sigma$ and $\mathrm{x}+3 \sigma$ abscissas is equal to $99.73 \%$ (where $\mathrm{x}$ is the average value).

In current practice, the control limit values with respect to the mean are precisely set equal to $\mathrm{x}+3 \sigma$ (UCL) and $\mathrm{x}-3 \sigma$ (LCL).

It should be noted that the line of the average value to be reported in the diagram may be, according to the intended use for the chart, that corresponding to the average of the experimentally detected xi values, or that of the expected specification value, $x$.

In the first case, the objective of the paper is to statistically examine the process, to see if only common causes act on it, which is the dispersion of the results and finally to check if there are any possible variations. When the goal is to ensure that the process reflects the project specifications, inside the bandwidth UCL-LCL, value corresponding to the allowable deviation must be reported.

The control lines are useful for immediately identifying the out-of-control situation of the process and the need to act with corrective actions and avoid out-of-specification points.

The warning lines, often also represented on the diagrams, are vice versa used to highlight the approaching risk situations of out of control; these lines are generally fixed at a distance equal to $+2 \sigma$ and $-2 \sigma$ from the central line.

When the data calculated by a subgroup lies between the warning line and the adjacent control line, it is good practice to observe the process to understand if a special cause has occurred or if there is a process of significant alteration of one of the influencing parameters.

$\mathrm{R}-\mathrm{Chart}$, to be used in conjunction with X-Chart, can also be accompanied by an indication of the value evaluated as the average of the measurements carried out on the subgroups, or by that of specification. In this chart, of course, only the upper control limit is indicated. The observation of the $\mathrm{R}$ chart is above all useful to check the extent of the dispersion in the process.

The traditional control charts, as previously introduced, are characterized by stationary control limits, set at $\pm 3 \sigma$, with respect to the mean of the considered parameter under observation. In this case, reference is made to a system of Cartesian axes, with the number of subgroups taken on the abscissa and the values of the parameter under observation on the ordinate, with particular attention to the average of the same subgroups.

The typical control chart, therefore, will appear as in figure 1.

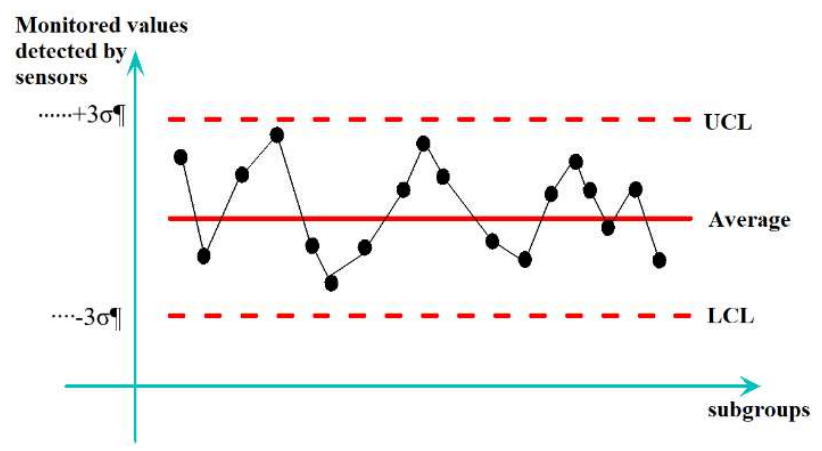

Figure 1. Control chart

In order to "transform" the traditional control chart into a "reliability chart" varying over time, it is necessary, as a first step, to make the abscissa axis linked to time, replacing the data collected on the abscissa of the subgroup data from the sensors according to their acquisition sequential time.

\subsection{Time variance monitoring chart (case $\sigma$ constant)}

For the purposes of the construction of the proposed monitoring reliability chart, assessed by the failure rate relating to the particular sensor of the specific geographical partition of the area to be monitored, the following assumptions must be observed:

- constant $\sigma$ variance: the characteristic under examination does not introduce a variation in the variance over time;

- approximately constant failure rate $\lambda$ : given the failure rate the reliability function $\mathrm{R}(\mathrm{t})$ will be known, which decreases exponentially with time (Toscano and Lyonnet, 2008) (Fig. 2). 


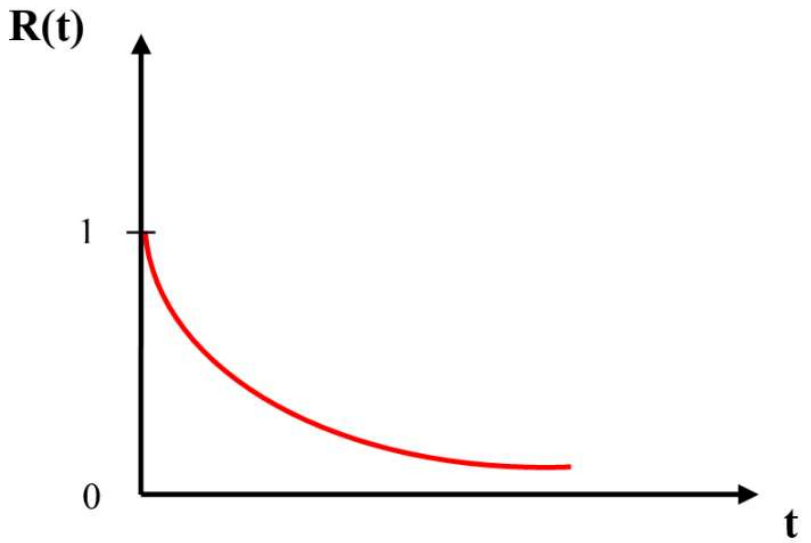

Figure 2. Reliability curve

On the basis of the hypotheses taken into consideration, the control chart changes by presenting variable limits, no longer of the type $\pm 3 \sigma$ (stationary), but of the type \pm ( $3 \mathrm{~K} \sigma$ $+k^{\prime} \sigma$ ), where the coefficient $k$ depends on time through the reliability function with $k=f[R(t)]$ and with coefficient $k$ to be considered as a design parameter representing the tolerance increase to be assigned to the monitoring system. The choice of $\mathrm{k}$ should consider that in the initial phase the reliability value is maximum and therefore the probability of non-compliance is characterized by low average.

In fact, in the modeling of the system, an increase of the tolerance equal to $3 \sigma$ of the additional quantity k' $\sigma$ has been assumed, which represents the largest share from which to start exponentially degrading the control limits.

Particularly, for a constant failure rate $\lambda$ it results as in (2).

$$
R(t)=e^{-\int_{0}^{t} \lambda(t) d t}=e^{-\lambda t}
$$

In $\mathrm{t}=0$, the reliability is maximum. Therefore, the constraint of a $3 \sigma$ tolerance can result in a high cost of managing the system (given by the sum of the cost of the control and the cost of restoring the system) in relation to the low probability of having an out of control. Therefore, it is more appropriate, with the aim to minimizing the cost / benefit ratio (objective ratio), to risk in this phase of being out of control, while having a lower system management cost.

In the following instants of time, vice versa, always with the aim to minimizing the objective ratio, it is more convenient, being the increased probability of finding an out of control, to adopt a more selective control in which the term $3 \sigma$ decreases as $\boldsymbol{e}^{-\lambda t}$.

Considering the incremental value $\mathrm{k}$ 's, an intersection point beyond which the control will be more selective than the stationary limit $3 \sigma$ will remain identified, depending from the failure rate of the system under observation and the $\sigma$ of the measured data.

With the same peculiar rules of a traditional control chart, but taking into account the hypotheses previously defined and the new variable control limits, it will be possible to construct the reliability control chart increasing severity of the control (Fig. 3).

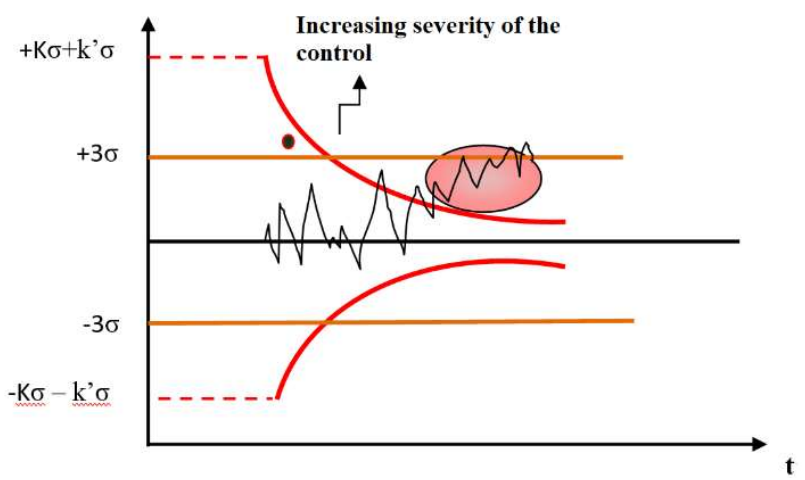

Figure 3. Time variance reliability control chart

The area highlighted in figure 3 shows an out of control situation detected by the time variance reliability control chart, but not by the traditional one. Once the reliability function for each geographical partition of an area to be monitored (hypothesis of homogeneous clusters per single partition) is known, therefore, the control becomes more strict (less wide control limits) where the decrease in the performance of the monitored characteristic becomes more evident.

Conversely, through the new time variance reliability control chart (TVRC2), however, it is possible to detect in the initial section a situation of out of control for the traditional chart, which is instead considered in control by the reliability chart. The chart is initially characterized by milder limits, with an aim to maximizing costs/benefits ratio. In the initial phase, in fact, the low probability of having an out of control does not justify corrective actions to improve the sensor networks. While in the final phase the high probability justifies a more selective control system. In fact, making the control more severe implies an increase in costs initially avoided through this proposed approach. It is useful to make the control dependent from the reliability function, according to which the network of remote sensors, during their useful life, works by reducing its performance only over time.

\subsection{Time variance monitoring chart (case $\sigma$ not constant)}

Indeed, the measurements acquired through a sensors' network could be affected by intrinsic variability deriving from degradation over time (Basso et al., 2009). In fact, there is an increase in the variance, $\sigma$ (caused both by the measurand and by the measurement system), relating to the different values progressively measured, as shown in figure 4. 


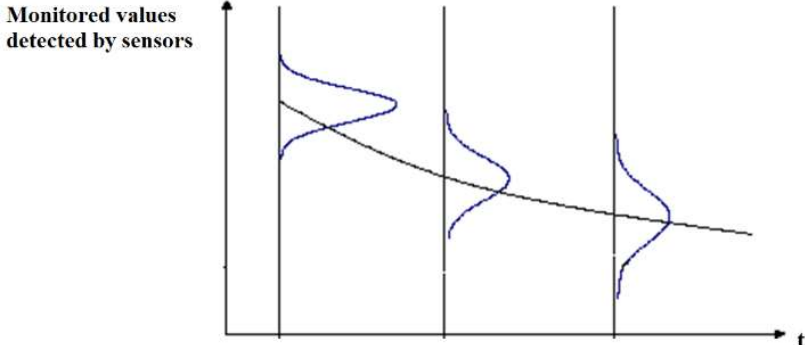

Figure 4. Intrinsic variability

It is possible to approximate the variance trend over time through a linear law such as $\sigma=\mathrm{a}_{1}+\mathrm{a}_{2} \mathrm{t}$.

In this case, the time variance reliability control chart will have control limits resulting from the envelope of parametric curves in increasing $\sigma$ (given the linear relationship), i.e. of greater intensity gradually over time (Fig. 5).

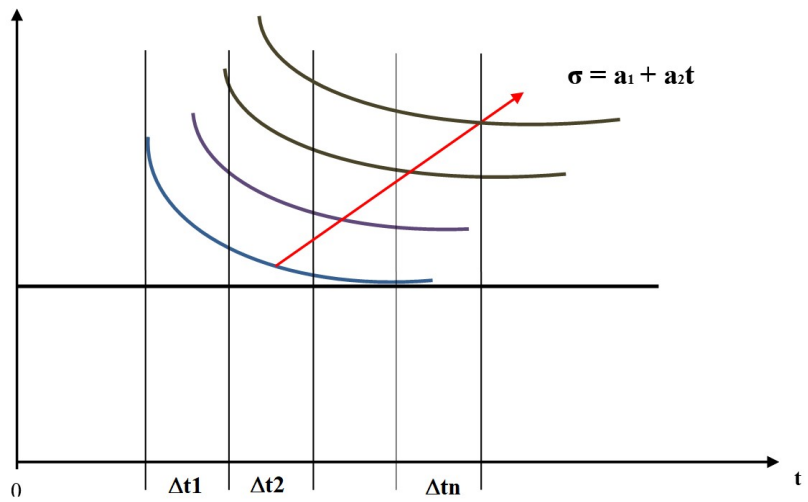

Figure 5. Case $\sigma$ not constant

Therefore, the previously defined hypothesis of constant variance over time should be removed (also if it can be considered valid approximately only for the first intervals used for taking the measurements of the various subgroups, for which the variance, in fact, does not present significant differences).

In this sense, suppose considering n elements that make up the subgroups in each of the 4 time intervals considered.

Generally, it can be said that for each time interval $\Delta \mathrm{t}_{\mathrm{i}}(\mathrm{i}=$ $1, \ldots 4)$, there is an increasing value of $\sigma_{i}(i=1, \ldots 4)$. This happens because the variance of the parameters under observation in the subgroups undergoes a degradation between one time interval and the next one. Therefore, it could possible to obtain a bundle of parametric limit curves in $\sigma$, parallel and translated according to the linear relationship $\sigma=\mathrm{a}_{1}+\mathrm{a}_{2} \mathrm{t}$, such that the control limit will be of the type $+\left(3 K \sigma_{i}+K^{\prime} \sigma_{i}\right)$.

The Upper Control Limit (UCL) to be considered is the union of the various branches of the $\sigma$-parametric beam,

\footnotetext{
${ }^{1}$ The monitoring activity was carried out in collaboration with the Measurement Laboratory of the "Mediterranea" University of Reggio Calabria, Italy, with Professor Claudio
}

obtained through the knowledge of the variation $\sigma(t)$ (Fig. 6).

Obviously, it is possible to iterate this construction also at the Lower Control Limit (LCL) of the reliability time variance monitoring chart.

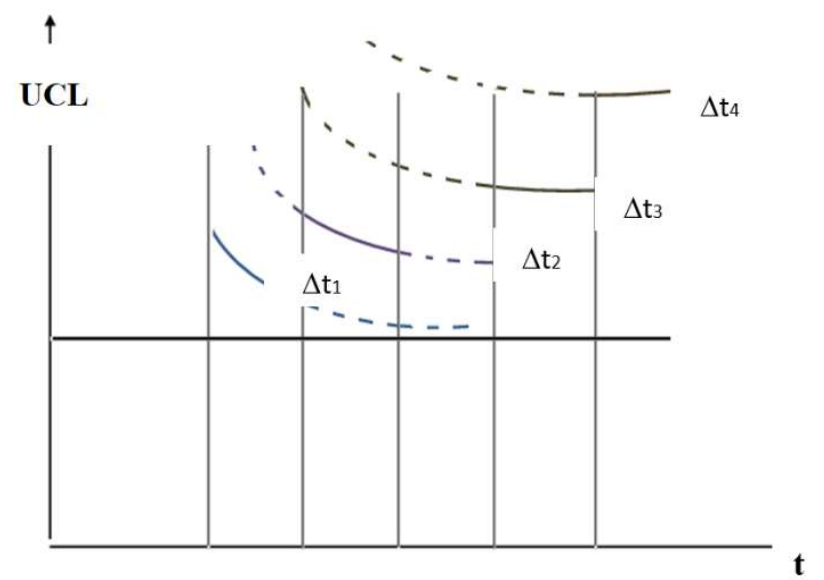

Figure 6. Envelope Upper Control Limit

\subsection{Spatial approach}

Once the model of temporal influence on the performance of the monitored environmental characteristics in an area is known, it is possible to move to a spatial approach and, therefore, to proceed to a space-time data fusion activity.

In fact, by considering homogeneous clusters of sensors in the geographical partitions into which a large area is divided, it is possible to obtain as many envelope-curves as there are geographical partitions.

In this way, great efficiency is achieved in remote sensing, minimizing the uncertainty on the data detected and maximizing the management costs of the sensor network.

At each point of a monitored area it is therefore possible to know the severity of the control to be set and it is possible to identify any anomalous situations of out of control without false positives or false negatives. The level of severity to set in each part of the monitored area will depend from its particular feature in term of human or artificial risk.

This generally doesn't happen in sensor networks that do not foresee neither a geographical partition in homogeneous areas, neither a temporal dependence on the uncertainty of the data collected.

\section{Application}

The proposed model was applied in a densely populated urban area ${ }^{1}$.

\subsection{Configurable area partitioning}

The number and size of the clusters are not constant but variable and configurable according to the needs (Zhang,

Maria De Capua (Chief of Laboratory) and Prof. Rosario Morello. 
Chen, 2019) and to the different types of risks as already highlighted. Care is paid about the determination of the single cluster in order to achieve a correlation among the different measurements within the local area; in fact it is a pressing constraint for a meaningful processing of data which must be comparable. Once selected a proper Cartesian coordinates system, the horizontal and vertical dimensions of the investigated area have to be selected. Then, by choosing the starting width of the grid, an initial mask indicates the location coordinates where the sensors have to be placed (Chen at al., 2014). Often is recognizable the need of analyzing particular areas more in depth, cause of the bigger population density or cause of the presence of electromagnetic pollution sources. So the implemented algorithm, once drawn the geographical grid, waits for one of this two inputs: a density of population function $\mathrm{f}(\mathrm{x}, \mathrm{y})$ of the examined zone or an indication of the sub-area, chosen by user, that has to be more precisely investigated. In the first case, the mean of the f function is evaluated, and the algorithm automatically thickens sensors in the zones where this mean overcomes a fixed threshold value. In the second case, the information about the zone where thicken the sensors is obtained through selection of the coordinates of a point in the sub-area or by clicking a point of the graph. The algorithm automatically thickens sensors in the sub-area grid, by dividing each zone in equal subzones (Fig. 7). User can choose to continue the thickness process, for a more narrow grid or to stop it. Through this algorithm, a simple, graphical information about the location where positioning the sensors can be achieved.

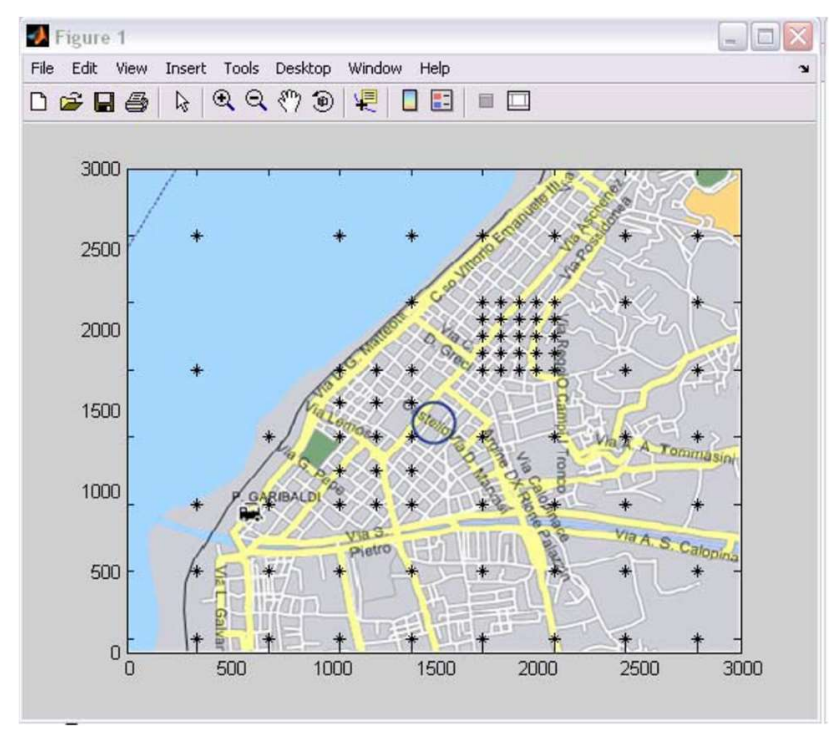

Figure 7. Configuration sensors' area partitioning

Wide urban area has to be monitored, so it is required a distributed architecture for the measurement system (He at al., 2016). The network design consists of wireless and smart web-sensors moving through the whole area (De Capua et al, 2005a,b). The sensing units and the same network have been projected according to the guidelines of the IEEE 1451 Standard (IEEE). In order to reduce the computational burden of each sensor, the area has been partitioned in several local zones. The specifications of the partition are function of the desired resolution and accuracy, so the number and size of the zones are variable according to the severity level of the sampling plan. Starting from information on the population density distribution and topographical data of the area, an original algorithm allows to perform the area partition. In details, at first, an initial grid is applied on the map of the area. According to the available resources and the sensors number, the user specifies the number of partitions. By a simple subdivision of the total territory extent, the area is equally divided in several local zones having the same size. Subsequently it is possible to thicken the partition in the 'sensible zones', which require a greater level of attention. Typically, a partition made up of numerous small zones should allow a more careful monitoring, but on the other side it would involve high costs.

So, the matter requires a reasonable compromise between the tolerable costs and the desired accurateness of the monitoring. In fact, the limitation of the available resources imposes a clear constraint to the maximum number of allowed zones in the area partition. So, it needs a criterion for characterizing the zones which require more attention and a more careful monitoring. A first parameter of choice is the population density. Densely populated zones have high risks in presence of hazardous situations, if the possible consequences on population are considered. Therefore, the algorithm reduces the size of the clusters characterized by high population density or by the presence of 'sensible targets' as hospitals or schools. The single interest zone is then further divided in other four additional sub-zones of same size (Fig. 8). In this way specific plans allow to perform a thorough monitoring of the sub-zones, in order to get more information depending on the chosen detail level. 


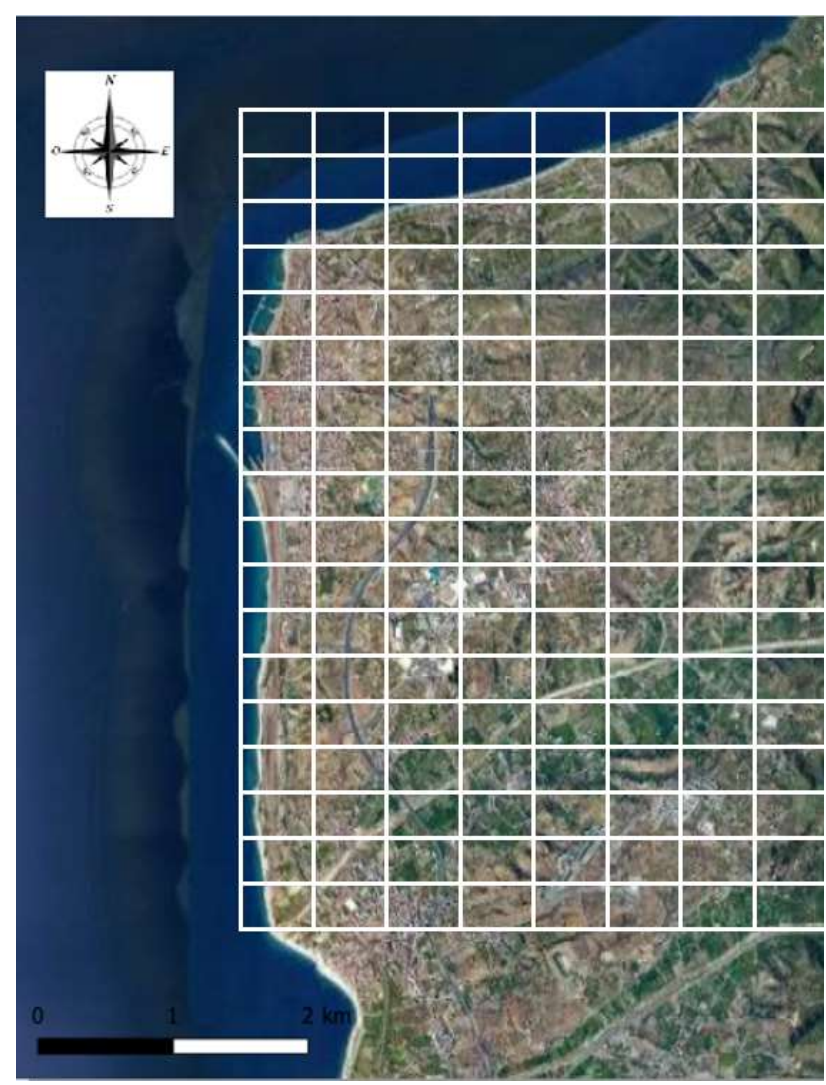

Figure 8. Partition of the monitored area.

The single web-sensor is equipped with probes measuring the electromagnetic field levels. The sensing unit (Fig. 9), has been realized by three transducers orthogonally displaced on a plexiglas support according to a Cartesian system x-y-z.
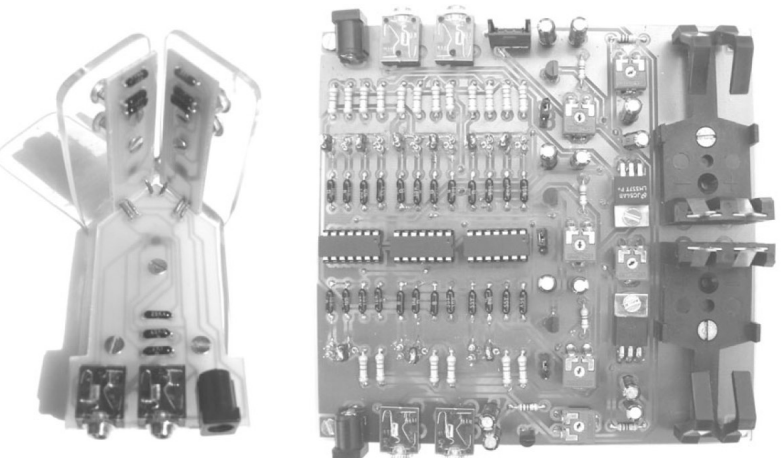

Figure 9. Sensing unit and conditioning circuit.

The output signals are analog voltages ranging from $\mu \mathrm{V}$ to $\mathrm{mV}$ values, each signal is proportional to the field orthogonally colliding with the surface of the device $(\mathrm{Hu}$ et al., 2016). By a conditioning circuit the three signals are amplified according to the working levels of the system. Several scales of measuring are available, so a set of programmable switches allows to perform an auto-scale setting. In this way the smaller scale is selected according to the input signal optimizing the resolution and accuracy of sensing. A 16 bit A/D converter digitalizes the three signals.

The control unit is based on a microcontroller architecture which manages the data-flow operations. So, according to the sampling frequency, the total electromagnetic field level, by the modulus of the three field levels, is stored in a Secure Digital (SD) memory which allows a sufficient autonomy of recording for several days. A General Packet Radio Service (GPRS) modem permits wireless communication, whereas a Global Positioning System (GPS) module provides the geographical location of the sensor. Two $9 \mathrm{~V}$ batteries provide the required voltage supply making the sensor an independent unit.

After the sampling procedures, each sensor sends acquired data to the own dynamic Web Page in Active Server Pages (ASP) format. Every page stores both the electromagnetic field levels of the single zone, and information on the same sensor about its history, metrological characteristics and operating state, as explained in the third paragraph. A remote workstation gains access to the Web pages information only by means of a password. In this way, it will have a first view on the field behaviour in each zone (Fig.10). Data are then processed with the time variance reliability control chart's procedure in order to test the electromagnetic field compliance with the exposure limits.

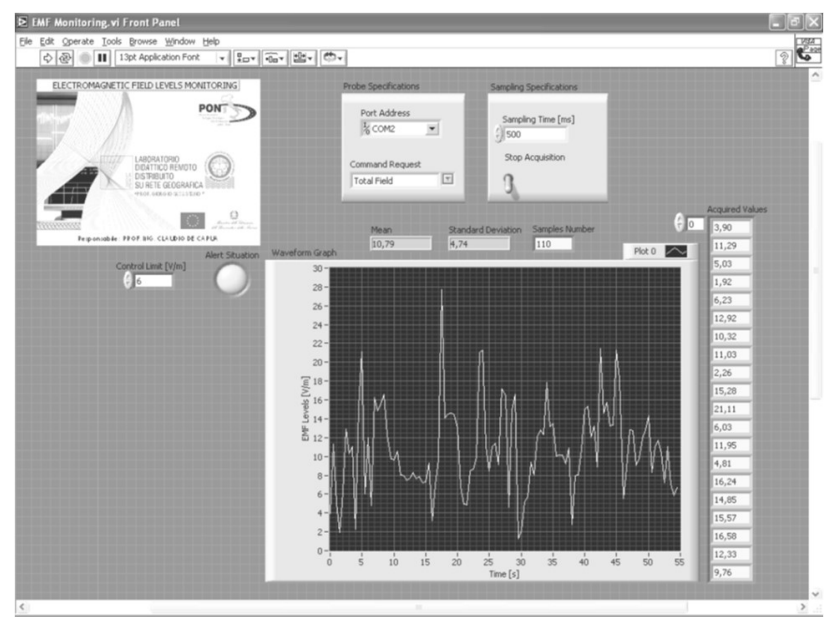

Figure 10. Data Acquisition Web Interface.

\subsection{Results}

The results considered $\sigma$ constant are shown in figure 11 .

From the comparison between the traditional chart and the TVRC2 one, there is initially an out of control not detected by the new chart. However, the low probability of having an out of control (as indicated by the reliability function), indicates that the point seen on the chart is an isolated point, and therefore the cost of a restore intervention on the system (in this case on the EM field sensor) it's not convenient.

On the contrast, in the tenth subgroup (the subgroups are ordered according to time as explained in the previous paragraphs) from the observation of the traditional chart, unlike the reliability one, a control situation is observed, 
where instead the system is reducing its performance, given the reliability function. Therefore, starting from the tenth subgroup onwards, all the effectiveness of the more selective proposed model is clear.

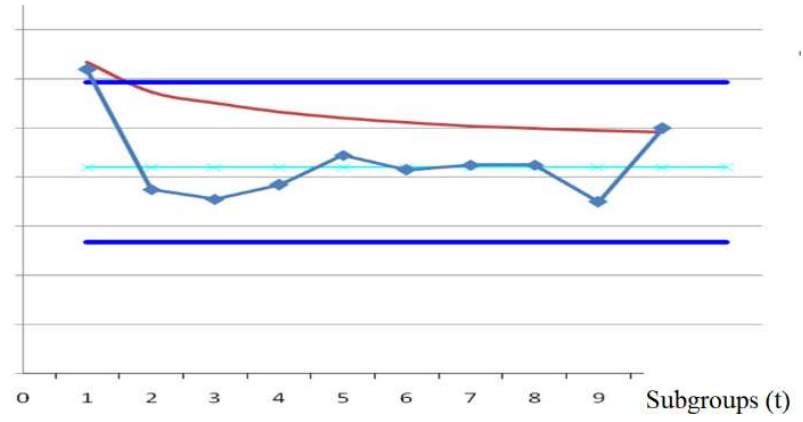

Figure 11. EM monitoring with TVRC2. Case $\sigma$ constant.

In figure 12 the particularization to the case $\sigma$ not constant with the beam of the parametric curves in $\sigma$, is shown.

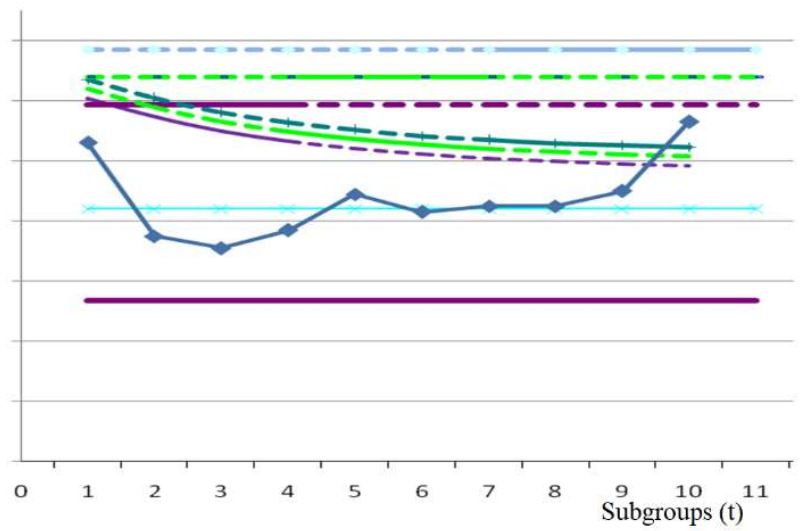

Figure 12. EM monitoring with TVRC2. Case $\sigma$ not constant.

\section{Conclusions}

In this paper has been proposed an innovative management of a sensor network for an environmental monitoring application.

The proposed model, starting from the partitioned configuration of a monitored geographic area, allows to take into account the different levels of degradation over time in the performance of the sensors associated with the different geographic partitions, progressively increasing the severity of the control of the monitored parameters. To this end, a modification of the traditional variable control chart is proposed, through the introduction of a reliability curve.

Particularly, the proposed modeling allows the use of a control chart for variables modified to adhere to real cases of degradation of the performance of the sensors of partitions of a geographical area to be monitored.

In fact, professionals have a double problem, on the one hand optimizing monitoring costs and on the other hand, having a very sensitive control tool. In order to satisfy both of these two antithetical needs, the reliability function has been used, as a "modulating guide" of the control limits with the result of having a milder control where conditions allow it (early monitoring periods), avoiding thus, especially in the case of automatic recovery systems, untimely and expensive interventions and, at the same time, to have greater sensitivity and greater severity in detecting anomalous events where conditions are more likely to occur (long monitoring periods).

The work reports the quantitative results of an application of the proposed modeling to a high-density urban area in which the levels of exposure to EM fields were monitored. Indeed, the theoretically described cases of greater efficiency of the new proposed time variance chart have been detected.

\section{References}

Basso, D., Pesarin, F., Salmas,o L., Solari, A. (2009. Permutation tests for stochastic ordering and ANOVA: theory and applications with R. London NY: Springer Verlag.

Chen, J., M. Deng, X. Mei, T. Chen, Q. Shao, and Hong, L. (2014). Optimal Segmentation of a High-Resolution Remote-Sensing Image Guided by Area and Boundary. International Journal of Remote Sensing, 35,6914-6939.

Chang, J.Y.; Ju, P.H. (2012). An efficient cluster-based power saving scheme for wireless sensor networks. Eurasip J. Wirel. Commun. Netw., 1-10.

De Capua C., De Falco S., Liccardo, A. Morello, R. (2005b). A Virtual Instrument for Estimation of Optimal Calibration Intervals by a Decision Reliability Approach, Proc. of IEEE VECIMS 2005b, Giardini NaxosTaormina, Italy.

De Capua, C., De Falco, S., Liccardo, A., Morello, R. (2005a). A Sensor Data Fusion Approach for Remote Sensing and Processing of EM Field Levels with a Configurable Area Partitioning, Proc. of IEEE CIMSA Giardini Naxos-Taormina, Italy.

Elhoseny, M., Yuan, X., Yu, Z., \& Mao, C. (2015). Balancing energy consumption in heterogeneous wireless sensor networks using genetic algorithm. IEEE Communications Letters, 19(12), 2194-2197

Favretto, A. (2018). Checking vegetation changes with remote sensing: The case of the Trieste province (NorthEast of Italy). Remote Sensing Applications, 1-10.

Haibin, Y., Peng, Z. and Ye, L. (2017). Intelligent wireless sensor network system, Science Press, pp. 8-11.

He, S., Shin, H.D., Zhang, J., Chen, J. and Sun, Y. (2016). Full-view area coverage in camera sensor networks: Dimension reduction and near-optimal solutions. IEEE Trans. Veh. Technol., 65(9), 7448-7461.

Hu, Y., Dong, M., Ota, K., Liu, A., and Guo, M (2016). Mobile target detection in wireless sensor networks with adjustable sensing frequency. IEEE Syst. J., 10(3), 11601171 . 
Huitian, Lu., Kolarik, W. J. and Lu, S.S. (2001). Real-time performance reliability prediction. IEEE Transactions on Reliability, 50(4), 353-357.

IEEE 1451, Smart Transducer Interface Standards.

Qing, L., Zhu, Q., \& Wang, M. (2006). Design of a distributed energy-efficient clustering algorithm for heterogeneous wireless sensor networks. Computer Communications, 29(12), 2230-2237.

Toscano, R., Lyonnet, P. (2008). On-Line Reliability Prediction via Dynamic Failure Rate Model. Reliability IEEE Transactions on, 57(3), 452-457.

Zhang, J., Chen, J. (2019). An adaptive clustering algorithm for dynamic heterogeneous wireless sensor networks. Wireless Netw 25, 455-470. https://doi.org/10.1007/s1 1276-017-1648-1

Zhou, H., Wu, Y., Hu, Y., \& Xie, G. (2010). A novel stable selection and reliable transmission protocol for clustered heterogeneous wireless sensor networks. Computer Communications, 33(15), 1843-1849. 